\title{
SOME REMARKS ON A PROGRESSIVE WAVE SOLUTION OF AN EQUATION IN A HETEROGENEOUS ELASTIC MEDIUM
}

\author{
Isao ONDA \\ Department of Mathematics and Physics, Faculty of Technology, \\ Gunma University, Kiryu, Gunma
}

(Received July 9, 1973; Revised October 2, 1973)

\begin{abstract}
An elastic wave equation in a heterogeneous medium is solved by means of the WKB method. If it refers to the connection formula around a turning point, a solution that formally expresses a progressive wave, $A(x) \cos \{\omega t-$ $k(x) x$, does not always satisfy the principle of energy conservation. The wave propagation with physical significance should be discussed using the structure of the heterogeneous medium intervening between two homogeneous half spaces.
\end{abstract}

\section{Introduction}

A reflected wave in a homogeneous medium is completely split off from the incident wave, in its formal expression. In contrast, in a heterogeneous medium no meaning can be attached, in general, to the wave field decomposed into two progressive components. ScHelkunoff (1951) explained this by stating that the field in a heterogeneous medium could be written formally as $A(x) \exp \{i \omega t-i \Phi(x)\}$ where $\Phi(x)$ is an increasing function of $x$, but it was not possible to split the expression uniquely into two progressive components. This can easily be understood from several papers on wave propagation in a periodic structure by RAYLEIGH (1887), YOSHIDA (1947), HoNMA and NAGAMUNe (1950), and YoshrYAMA and ONDA (1962). This also is interpreted as follows: A heterogeneous medium can be approximated by a suitable layered structure of many homogeneous media. Thus waves are propagated with apparent attenuation due to generation of reflected waves at each interface, as they pass through that layered structure. This means that a mathematical solution of the wave equation in a heterogeneous medium does not always correspond to that of an individual progressive wave. This kind of the comment is found in the field of water waves passing over a bottom of variable depth (Kajiura, 1961).

This paper concerns with the wave separation in a more general hetero- 
geneous medium, discussed by means of the phase-integral (Jeffrey's or WKB) method. This method is of importance in many physical applications, such as quantum mechanies and electromagnetic wave propagation (LANGER, 1937; HEADING, 1962; BAHAR, 1967).

\section{Solution of Wave Equation with Physical Significance}

For simplicity, propagation of plane harmonic waves in an isotropic, heterogeneous medium where the density $\rho$ and the modulus of elasticity $E$ vary as continuous functions of the one spatial coordinate $x$ is considered. The displacement of the wave, $u$, is satisfied by the equation

$$
\frac{\partial}{\partial x}\left(E \frac{\partial u}{\partial x}\right)+\rho \omega^{2} u=0
$$

where $\omega$ is the angular frequency of the wave. Following Yosmiyam (1960), the independent variable is changed from $x$ to the travel time of the ray $\tau$, and Eq. (1) yields

where

$$
\mathrm{d}^{2} \varphi / \mathrm{d} \tau^{2}+\left(\omega^{2}-\alpha^{2}\right) \varphi=0
$$

and

$$
\left.\begin{array}{c}
\varphi=(\rho c)^{1 / 2} \cdot u, \quad c=(E / \rho)^{1 / 2}, \quad \tau=\int^{x} c^{-1} \mathrm{~d} x \\
\alpha^{2}=-(c / \rho)^{1 / 2}(\mathrm{~d} / \mathrm{d} x)\left\{\left(\rho c^{2}\right)(\mathrm{d} / \mathrm{d} x)(\rho c)^{-1 / 2}\right\} .
\end{array}\right\}
$$

Wave propagation is discussed through the solution of an ordinary differential Eq. (2). Equation (2), however, can explicitly be solved only in a few cases of $E$ and $\rho$ variations. One object of this paper is to derive some important characters of solution of Eq. (2). any:

The following assumptions are valid physically for a progressive wave, if

(i) The half space in the negative $x$ is homogeneous,

$$
\tau=x / c_{0} ; \quad \text { and } \quad \alpha^{2}=0 \text { for } x \leq 0,
$$

(ii) the displacement and thus $\varphi$ are bounded throughout the whole medium,

$$
|u|<u_{0}, \quad \text { or } \quad|\varphi|<\varphi_{0}, \quad \text { for } 0 \leq \tau<\infty .
$$

where $c_{0}, u_{0}$, and $\varphi_{0}$ are constant.

If the variation of $\alpha^{2}$ is small, approximate solutions of Eq. (2) will be written in such a permissible form as follows: In case $\omega^{2}-\alpha^{2}>0$,

$$
\varphi_{1}^{\prime} \sim \Omega^{-1 / 2} \cdot\left[A_{1} \cos \left(\int \Omega \mathrm{d} \tau\right)+B_{1} \sin \left(\int \Omega \mathrm{d} \tau\right)\right]
$$


or

$$
\varphi_{2} \sim \Omega^{-1 / 2} \cdot\left[A_{2} \exp \left(i \int \Omega \mathrm{d} \tau\right)+B_{2} \exp \left(-i \int \Omega \mathrm{d} \tau\right)\right]
$$

and in case $\omega^{2}-\alpha^{2}<0$,

or

$$
\varphi_{8} \sim \Omega^{-1 / 2} \cdot\left[A_{8} \cosh \left(\int \Omega \mathrm{d} \tau\right)+B_{3} \sinh \left(\int \Omega \mathrm{d} \tau\right)\right]
$$

$$
\varphi_{4} \sim \Omega^{-1 / 2} \cdot\left[A_{4} \exp \left(\int \Omega \mathrm{d} \tau\right)+B_{4} \exp \left(-\int \Omega \mathrm{d} \tau\right)\right]
$$

where

$$
\Omega=\sqrt{\left|\omega^{2}-\alpha^{2}\right|}
$$

and the $A$ 's and $B$ 's are constants determined from the initial and boundary conditions. In these formal solutions, one satisfying the second assumption (5) is of the type (9) with $A_{4}=0$ :

$$
\varphi \sim B \cdot \Omega^{-1 / 2} \cdot \exp \left(-\int \Omega \mathrm{d} \tau\right) \quad \text { for } \quad \omega^{2}-\alpha^{2}<0, \tau \rightarrow \infty .
$$

For a homogeneous medium or such a heterogeneous one as $(\rho c)^{8 / 2}=a+$ $b \cdot \int \rho(x) \mathrm{d} x, a$ and $b$ being constant, the factor $\alpha^{2}$ vanishes. If a medium is neither of them, $\alpha^{2}$ has a positive value independent of frequency $\omega$. Frequency $\omega$ is taken into account for all the real values, and thus there exists one frequency, at least, which satisfies $\omega^{2}-\alpha^{2}=0$. Equation (11) valid for $\omega^{2}-\alpha^{2}<0$ must be continued to a solution valid for another region by means of a connection formula (e.g., HEADING, 1962, p. 80). Without loss of generality, the solution continued from Eq. (11) to the other region is written as

$$
\varphi \sim A \cdot \Omega^{-1 / 2} \cdot \sin \left(\int \Omega \mathrm{d} \tau+\pi / 4\right), \quad A=2 B, \quad \text { for } \quad \omega^{2}-\alpha^{2}>0,
$$

whence

$$
u \sim A \cdot \Omega^{-1 / 2}(\rho c)^{-1 / 2} \cdot \sin \left(\int \Omega \mathrm{d} \tau+\pi / 4\right) \exp (i \omega t) .
$$

The solution (13) is the only solution with physical significance of waves in a heterogeneous medium, and it is regarded as a standing wave. In other words, waves in a heterogeneous medium cannot separately be expressed as progressive and retrogressive waves, Another exceptional heterogeneous medium, in which two independent solutions decide the field in progressive and retrogressive waves is of a linearly dependent variation in velocity (YOSHIYAMA, 1960).

The stability and partial reflection of the progressive wave should be discussed using the transmission and reflection coefficients in the structure with the heterogeneous medium under consideration between two homogeneous 


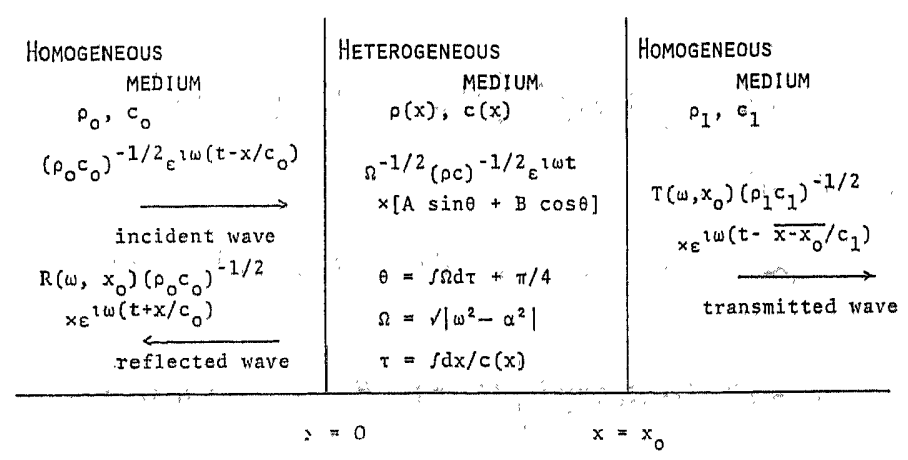

Fig. 1. Model used for wave propagation in a heterogeneous medium.

media in one of which an original wave is incident (see Fig. 1). In this case it is assumed that the velocity is continuous at each boundary, in order to eliminate the effect of boundary reflections (ONDA, 1965).

\section{Solution of a Progressive Wave Expression}

The formal solution of a progressive wave like type (7) was interpreted by the help of the geometric-optical approximation by BREMMER (1951): A hoterogeneous medium is replaced by a multi-layered structure each layer of which is homogeneous. Only a wave passing across each interface in the $+x$ direction is calculated, and thus all the reflections arising at each interface are neglected. Last, the thickness of each layer and the difference of wave numbers between adjacent layers are made to tend to infinitesimal together. The result thus obtained for $\omega \gg|\alpha|$ is written as

$$
u_{p}(x)=u_{p}(0) \sqrt{\frac{\rho(0) \cdot c(0)}{\rho(x) \cdot c(x)}} \exp \left(-i \int_{0}^{x} \frac{\omega}{c(x)} \mathrm{d} x\right)
$$

where $u_{p}(x)$ and $u_{p}(0)$ are the progressive waves at $x=x$ and $x=0$, respectively. In expression (14), it should be noted that the multiple reflection in a stratified medium is not taken into account.

Consequently, the variation of the initial phase of a wave train such as the first arrivals of longitudinal and shear waves is correctly understood by Eq. (14), but if the change of wave forms is estimated through Eq. (14), it may lead to an improper conclusion.

\section{REFERENCES}

BAHAR, E., Generalized WKB method with applications to problems of propagation in nonhomogeneous media, J. Mathematical Phys., 8, 1735-1746, 1967.

BREMMER, H., The W.K.B. approximation as the first term of a geometric-optical series, Comm. Pure Appl. Math.; 4, 105-115, 1951. 
Heading, J., An Introduction to Phase-Integral Methods, Methuen, 1962.

Honma, S., and T. Nagamune, Kyôkai-sô ni okeru hadô no hansya ni tuite, Quart. J. Seism., 14, 47-55, 1950 (in Japanese).

JEFFREYS, H., On the use of asymptotic approximations of Green's type when the coefficient has zeros, Proc. Cambr. Phil. Soc., 52, 61-66, 1956.

KAJIURA, K., On the partial reflection of water waves passing over a bottom of variable depth, IUGG monograph, no. 24 ( $X$-th Pacific Science Congress, Tsunami Symposium), 206-230, 1961.

LANGER, R. E., On the connection formulas and the solutions of the wave equation, Phys. Rev., 51, 669-676, 1937.

ONDA, I., Effects of intervenient boundaries on the elastic wave propagation, Bull. Earthq. Res. Inst., 43, 1-14, 1965.

RAYLEIGH, LORD, On the maintenance of vibrations by forces of double frequency, and on the propagation of waves through a medium endowed with a periodic structure, Phil. Mag., 24, 145-159, 1887.

SChel Kunoff, S. A., Remarks concerning wave propagation in stratified media, Comm. Pure Appl. Math., 4, 117-128, 1951.

YoshIDA, K., On the forced waves in a canal of rapidly varying section, Geophys. Notes, Fac. Sci., Tokyo Univ., no. 30, 1947.

YoshryAmA, R., Stability of waves through a heterogeneous medium and apparent internal friction, Bull. Earthq. Res. Inst., 38, 467-478, 1960.

Yoshryama, R., and I. ONDA, Stability of waves through a heterogeneous medium and apparent internal friction, Part 2, with special remarks on wave propagation in a periodic structure, Bull. Earthq. Res. Inst., 40, 391-398, 1962.

WARE, J. A., and K. AKI, Continuous and discrete inverse-scattering problems in a stratified elastic medium. I. Plane waves at normal incidence, J. Acoust. Soc. Amer., 45, 911-921, 1969. 